Supporting Information for

\title{
Covalent Functionalized Black Phosphorus Greatly Inhibits \\ Nonradiative Charge Recombination: A Time-Domain ab Initio \\ Study
}

\author{
Yaqing Wei, ${ }^{1}$ Wei-Hai Fang, ${ }^{1}$ Run Long ${ }^{1 *}$ \\ ${ }^{1}$ College of Chemistry, Key Laboratory of Theoretical \& Computational
}

Photochemistry of Ministry of Education, Beijing Normal University, Beijing, 100875,

$$
\text { P. R. China }
$$

Here, we consider charge trapping and recombination dynamics in the pristine black phosphorus (BP), and the BP passivated with phenyl and nitrophenyl systems. The basis setting, kinetic equations and their solutions are presented below.

\footnotetext{
* Corresponding Author Email: runlong@bnu.edu.cn
} 
1. Pristine BP. The nonradiative electron-hole recombination takes places across the conduction band minimum (CBM) and the valence band maximum (VBM). The CBM and VBM correspond to the first excited state (ES) and ground state (GS), respectively. The Fermi level $\left(E_{f}\right)$ is identified with the blue dashed line. Coupled kinetic equations:

$$
\begin{aligned}
& \frac{d[E S]}{d t}=-k[E S] \\
& \frac{d[G S]}{d t}=k[E S]
\end{aligned}
$$

Solutions for the set of equations are:

$[E S]=e^{-k * t}$

$[G S]=1-e^{-k * t}$

Here, $k$ is the rate constant for quantum transition between the CBM and VBM. It is presented in Table 1.
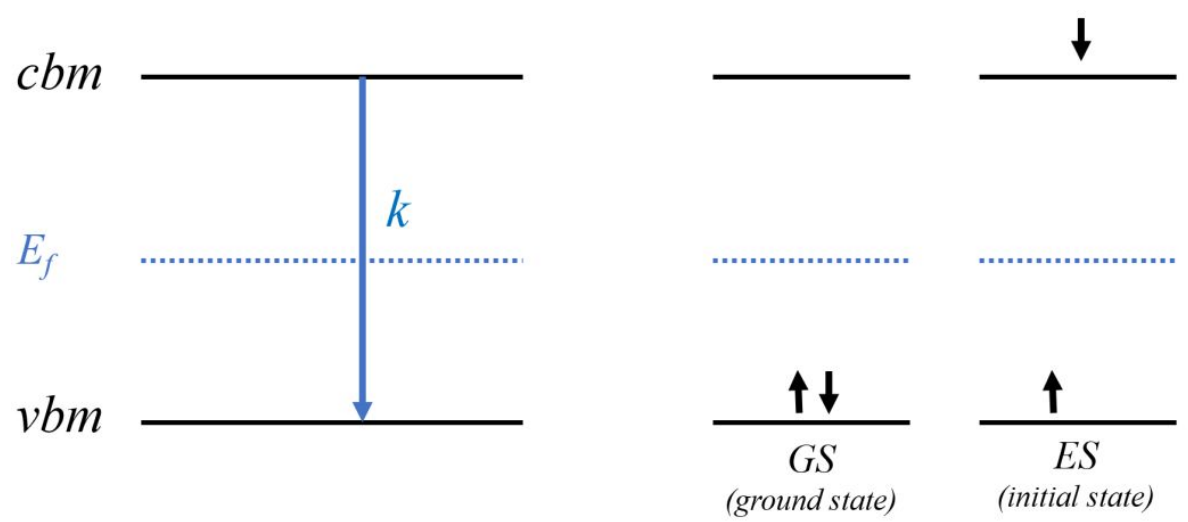

Figure S1. The schematic of electron-hole recombination pathway in the pristine BP. 
2. BP passivated with Phenyl and Nitrophenyl. The electron-hole recombination is assisted with either hole trap (spin-up channel) or electron trap (spin-down channel).

(a) Spin-up channel.

Coupled kinetic equations:

$\frac{d[E S]}{d t}=-\left(k_{2}+k_{3}\right)[E S]$

$\frac{d[\text { trap }]}{d t}=k_{2}[E S]-k_{1}[$ trap $]$

$\frac{d[G S]}{d t}=k_{3}[E S]+k_{1}[$ trap $]$

Solutions for the set of equations are:

$[E S]=e^{-\left(k_{2}+k_{3}\right) * t}$

$[$ trap $]=\frac{k_{2}}{\left(k_{2}+k_{3}-k_{1}\right)}\left(e^{-k_{1} * t}-e^{-\left(k_{2}+k_{3}\right) * t}\right)$

$[G S]=1-\frac{1}{\left(k_{2}+k_{3}-k_{1}\right)}\left\{k_{2} * e^{-k_{1} * t}-\left(k_{1}+k_{3}\right) * e^{-\left(k_{2}+k_{3}\right) * t}\right\}$

Here, $k_{1}, k_{2}, k_{3}$ denotes the rate constant for the quantum transition between pairs of electronic states of (CBM-trap), (trap-VBM), and (CBM-VBM), respectively. They are listed

in Table

1.
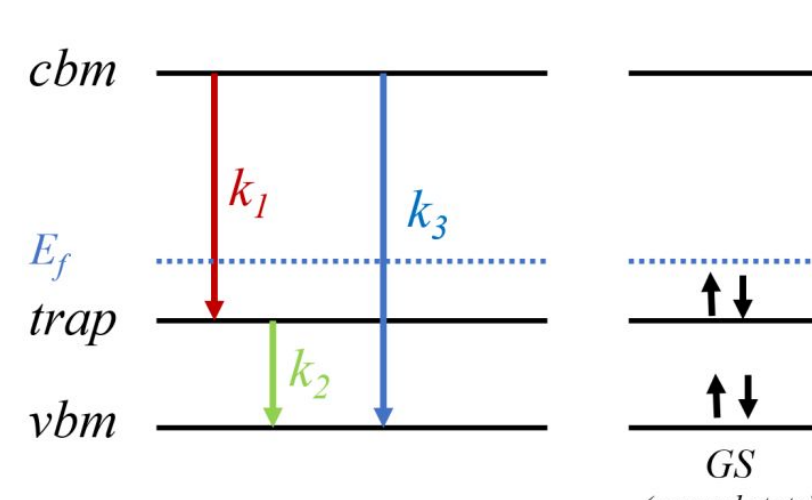

(ground state)
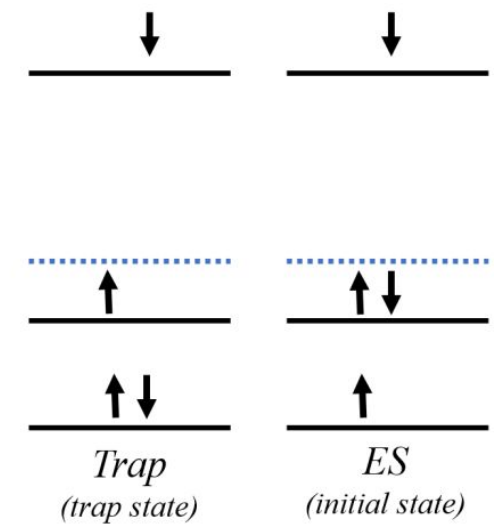
Figure S2. The schematic of hole trap assisted electron-hole recombination in the pristine BP passivated with phenyl and nitrophenyl systems in the spin-up channel. 
(b) Spin-down channel.

Coupled kinetic equations:

$\frac{d[E S]}{d t}=-\left(k_{1}+k_{3}\right)[E S]$

$\frac{d[\operatorname{trap}]}{d t}=k_{1}[E S]-k_{2}[\operatorname{trap}]$

$\frac{d[G S]}{d t}=k_{3}[E S]+k_{2}[\operatorname{trap}]$

Solutions for this set of equations are:

$[E S]=e^{-\left(k_{1}+k_{3}\right) * t}$

$[\operatorname{trap}]=\frac{k_{1}}{\left(k_{1}+k_{3}-k_{2}\right)}\left(e^{-k_{2} * t}-e^{-\left(k_{1}+k_{3}\right) * t}\right)$

$[G S]=1-\frac{1}{\left(k_{1}+k_{3}-k_{2}\right)}\left\{k_{1} * e^{-k_{2} * t}-\left(k_{2}+k_{3}\right) * e^{-\left(k_{1}+k_{3}\right) * t}\right\}$

Here, $k_{1}, k_{2}, k_{3}$ denote the rate constant for the quantum transition between pairs of electronic states of (CBM-trap), (trap-VBM), and (CBM-VBM), respectively. They are listed in Table S1.
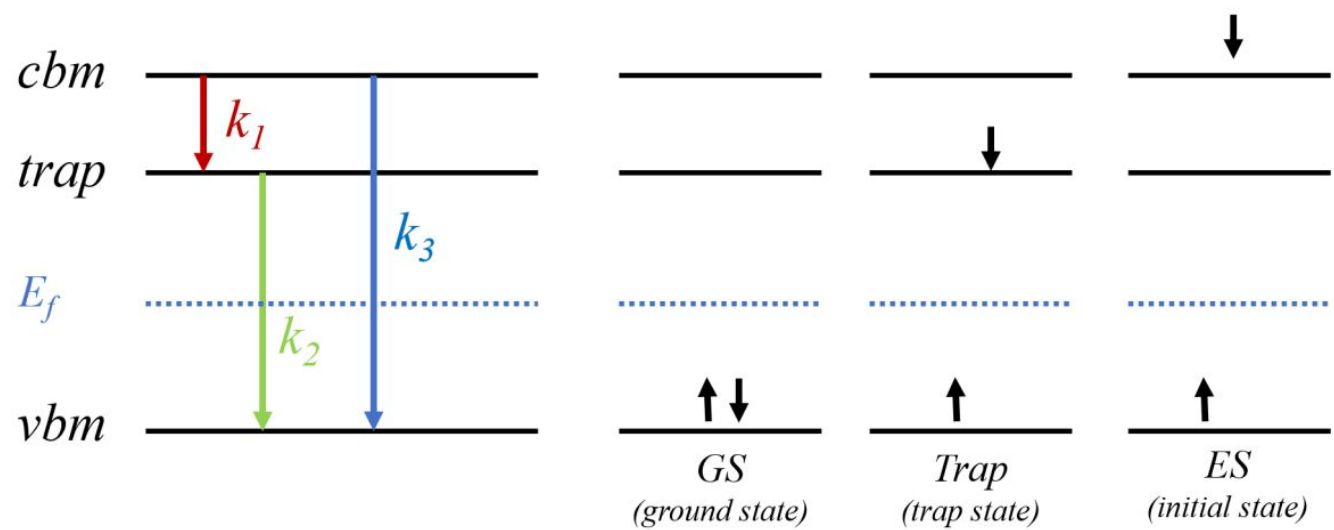

Figure S3. The schematic of electron trap assisted electron-hole recombination pathway in the pristine BP passivated with phenyl and nitrophenyl in the spindown channel. 
(a) Pristine

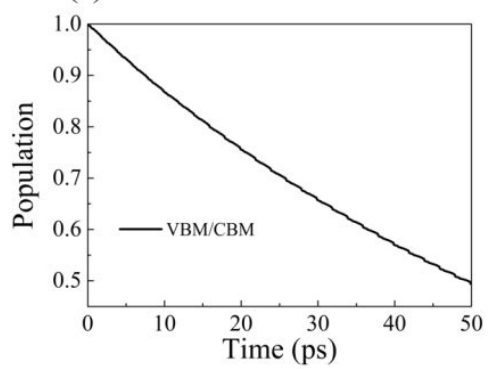

(b) Phenyl

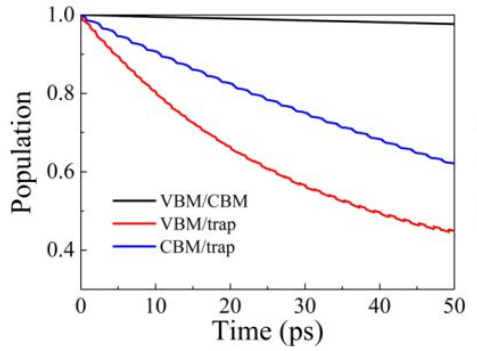

(c) Nitrophenyl

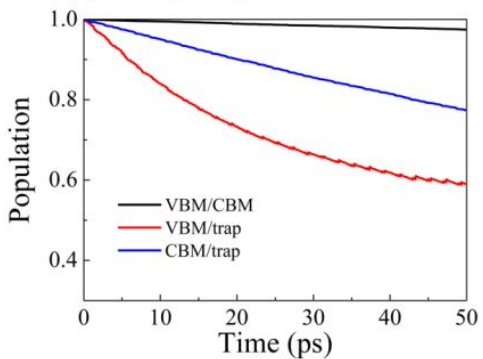

Figure S4. Time evolution of the interested state population in the spin-up channel of (a) pristine BP and BP passivated with (b) phenyl and (c) nitrophenyl. The calculated rate constants are summarized in Table 1. 
(a) Pristine

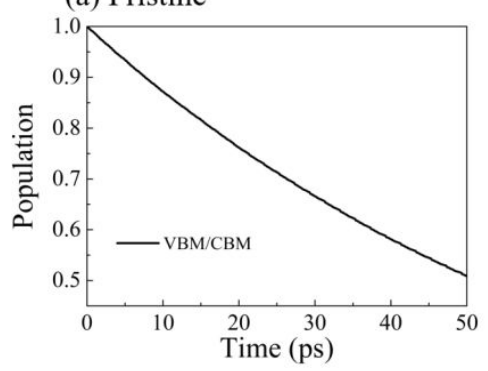

(b) Phenyl

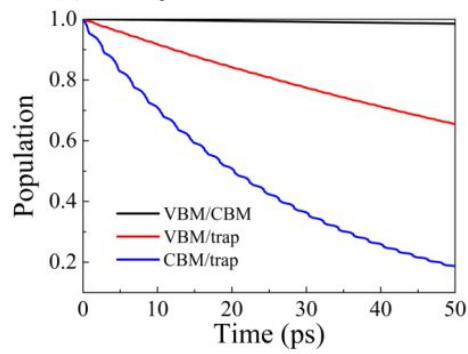

(c) Nitrophenyl

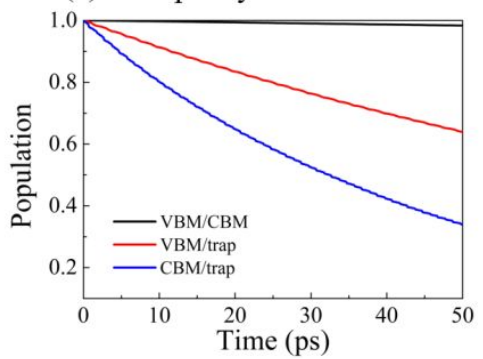

Figure S5. Time evolution of the interested state population in the spin-down channel of (a) pristine BP and BP passivated with (b) phenyl and (c) nitrophenyl. The calculated rate constants are summarized in Table S1. 
(a) Pristine

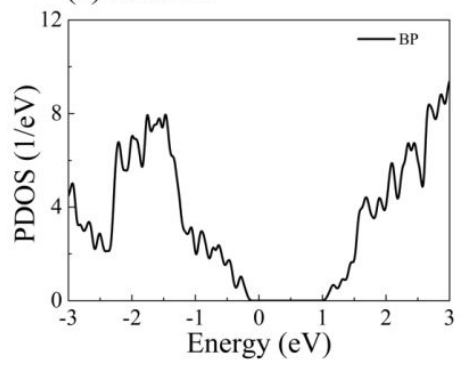

(b) Phenyl

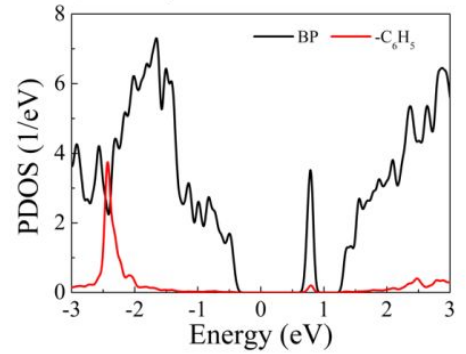

(c) Nitrophenyl

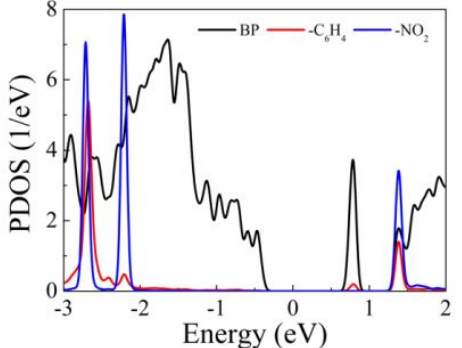

Figure S6. Projected density of states (PDOS) in the spin-down channel of (a) pristine BP, and BP passivated with (b) phenyl and (c) nitrophenyl systems. The zero energy is set to Fermi level. 


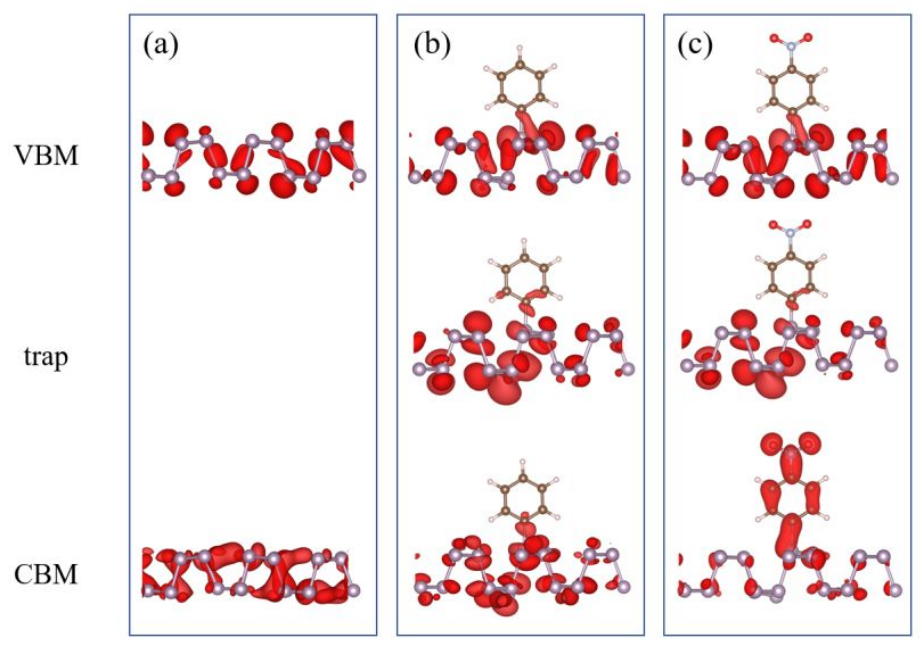

Figure S7. Spin-down charge density of key orbitals of (a) pristine BP, BP passivated with (b) phenyl and (c) nitrophenyl. The final state VBM, hole trap state, and initial state CBM for each system are presented respectively. Adsorbed molecules perturb charge density mixing and affect charge dynamics. 
(a) Pristine

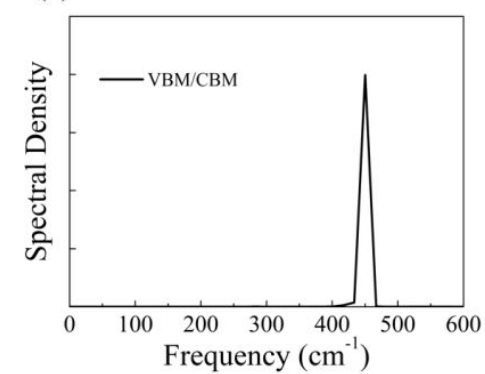

(b) Phenyl

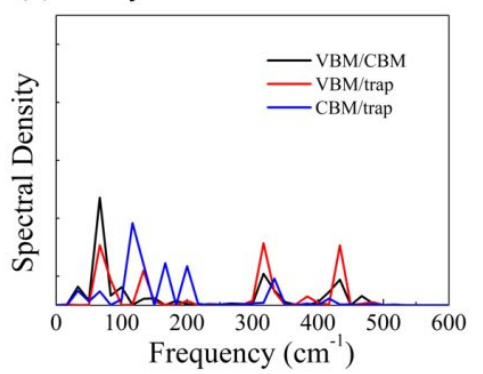

(c) Nitrophenyl

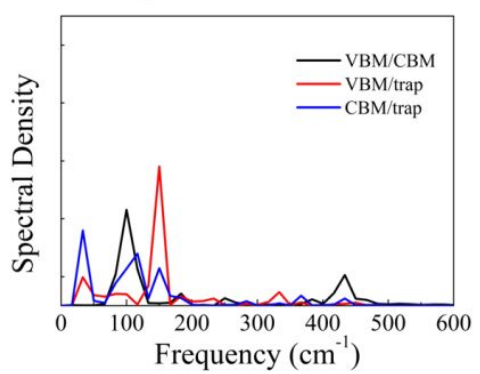

Figure S8. Spectral densities of (a) pristine BP, and pristine BP passivated with (b) phenyl and (c) nitrophenyl, computed using Fourier transform of energy gap fluctuation of each system, which characterize the vibrational modes participating into electron trapping and electron-hole recombination in the spindown channel. 
(a) Pristine

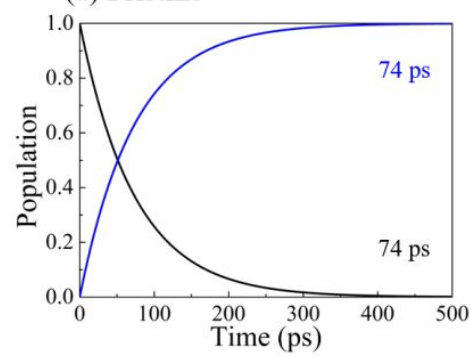

(b) Phenyl

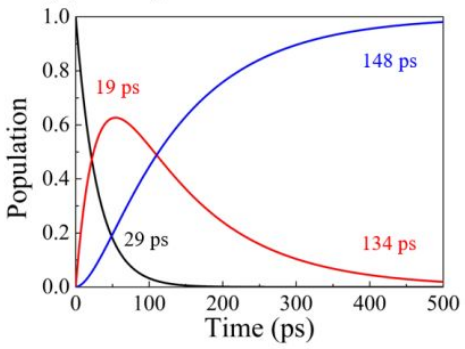

(c) Nitrophenyl

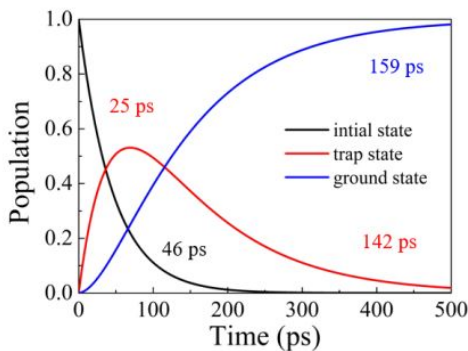

Figure S9. Electron trap assisted recombination dynamics in the spin-down channel for (a) pristine BP, BP passivated with (b) phenyl and (c) nitrophenyl. In the two passivated systems, the recombination occurs either between the conduction band free electrons and valence band free holes bypassing the electron trap state or between the trapped electrons and valence band free holes. Recombination in both pathways is delayed by a factor of 2-3. 
Table S1. Energy Gap, Pure-Dephasing Time, Average Absolute NA Coupling and Transition Rate Constant for Pairs of Electronic States in the Spin-Down Channel of the Pristine BP, and BP Passivated with Phenyl and Nitrophenyl Systems.

\begin{tabular}{|c|c|c|c|c|c|}
\hline & & Energy Gap & Dephasing & NA Coupling & Rate \\
\hline & & $(\mathrm{eV})$ & (fs) & $(\mathrm{meV})$ & $\left(\mathrm{ps}^{-1}\right)$ \\
\hline Pristine & VBM/CBM & 1.36 & 3.1 & 2.41 & 0.01355 \\
\hline \multirow{3}{*}{ Phenyl } & VBM/CBM & 1.79 & 6.3 & 1.24 & $2.95 \mathrm{E}-04$ \\
\hline & $\mathrm{VBM} /$ trap & 1.12 & 4.4 & 2.32 & 0.00846 \\
\hline & $\mathrm{CBM} /$ trap & 0.67 & 7 & 3.46 & 0.03397 \\
\hline \multirow{3}{*}{ Nitrophenyl } & VBM/CBM & 1.8 & 8.1 & 1.38 & $3.20 \mathrm{E}-04$ \\
\hline & VBM/trap & 1.11 & 4.7 & 2.47 & 0.00896 \\
\hline & $\mathrm{CBM} /$ trap & 0.69 & 4.6 & 2.8 & 0.02161 \\
\hline
\end{tabular}

